\title{
Ewing's sarcoma: A case report of a 52-year-old woman with recurrent tumor and literature review
}

\author{
WASEEM KHALIQ ${ }^{1}$, MOHAMMAD FARSHAD BAHADOR ${ }^{2}$, THOMAS NICHOLS LAURENCE ${ }^{3}$, \\ RONALD ANDREW SAPIENTE ${ }^{4}$, JAMES SHERIDAN LEWIS $\mathrm{Jr}^{5}$ and DAVID LEE GRAHAM ${ }^{4}$ \\ ${ }^{1}$ Department of Medicine, Johns Hopkins University School of Medicine, Baltimore, MD; ${ }^{2}$ University of Missouri, \\ Columbia, MO; ${ }^{3}$ Department of Internal Medicine, University of Illinois College of Medicine; \\ ${ }^{4}$ Department of Radiation Oncology, Carle Cancer Center/Mills Breast Cancer Institute, Urbana, IL; \\ ${ }^{5}$ Department of Pathology and Immunology, Washington University in St. Louis, MO, USA
}

Received January 18, 2011; Accepted September 26, 2011

DOI: $10.3892 / \mathrm{ol} .2011 .440$

\begin{abstract}
Ewing's sarcoma is the second most common primary sacral tumor. Ewing's sarcomas are rare, aggressive tumors with a tendency towards recurrence following resection and early metastasis. Although peak incidences are between the ages of 10 and 20 years, patients of younger or older age account for almost $30 \%$ of the cases. We report the case of a 52-year-old healthy female who presented with a 2-week history of pain in her right posterior thigh that was unable to be relieved by non-steroidal anti-inflammatory medicine and physical therapy. Magnetic resonance imaging demonstrated an irregular right presacral mass and core needle biopsy revealed a small, round blue cell neoplasm. Staging workup was normal and an open biopsy was positive for the ES translocation (22q12). The patient was treated with 17 cycles of vincristine, adriamycin and cytoxan with mesna rescue, alternating with ifosfamide and etoposide in addition to external beam radiation. Post-treatment imaging demonstrated complete resolution of the tumor. Six weeks post-treatment the patient presented with a recurrent tumor. This case emphasizes the importance of timely establishment of initial diagnosis, early metastasis in treatment responsive patients and under-utilization of positron emission tomography-computed tomography (PET-CT) during the treatment to detect sub-clinical metastasis.
\end{abstract}

\section{Introduction}

Ewing's sarcoma (ES) is the second most common bone tumor after osteosarcoma in children and adolescents. ES are aggressive tumors with a tendency towards recurrence following resection and pronounced proclivity toward early

Correspondence to: Dr Waseem Khaliq, Instructor of Medicine, Johns Hopkins University School of Medicine, 5200 Eastern Avenue, MFL West 6th Floor, Baltimore, MD 21224, USA

E-mail:khaliqmd@gmail.com

Key words: leg pain, Ewing's sarcoma, microscopic metastases, multimodal therapy hematogenous metastases to lungs and bone. No hereditary or congenital syndromes, environmental or known risk factors have been associated with the occurrence of ES. In $90 \%$ of cases, Ewing's sarcoma family tumor (ESFT) cells harbor the translocation $\mathrm{t}(11 ; 22)(\mathrm{q} 24 ; \mathrm{q} 12)$, and in the remaining $10 \%$ the variant translocation is $\mathrm{t}(21 ; 12)(22 ; 12)(1,2)$. Although peak incidence occurs between the ages of 10 and 20 years, patients of younger or older ages account for almost $30 \%$ of the cases (3). Poor prognostic factors include tumor $\geq 8 \mathrm{~cm}$, pelvic primary, presence of metastases and age $>15$ at the time of diagnosis (4). Older patients with sarcoma also have a higher risk of thromboembolism (5).

\section{Case report}

A 52-year-old healthy female presented with a 2-week history of pain in her right posterior thigh. The pain originated in the patient's right buttock and radiated to the back of the knee without a radicular component. Motor strength, sensory function and reflexes were normal. Musculoskeletal examination was within normal limits without tenderness of the hips or back.

Despite the use of non-steroidal anti-inflammatory medications and physical therapy the pain persisted. On subsequent reassessment, a radicular component was present. Magnetic resonance imaging (MRI) demonstrated an irregular-shaped right presacral mass with heterogeneous short $\mathrm{T} 1$ inversion recovery (STIR) hyperintensity (Fig. 1A) and T1 hypointensity (Fig. 1B). Additional evaluation with computed tomography (CT) of the pelvis revealed a complex mass with central necrosis and a thick enhancing wall extending into the right S2 neural foramen with lytic bone destruction (Fig. 1C) measuring $6 \times 6 \times 5 \mathrm{~cm}$ with compression of the right piriformis muscle posteriorly (Fig. 1D). No adenopathy or correlation to gynecologic structures of the pelvis were found.

An initial core needle biopsy of the mass revealed a small, round blue cell neoplasm, suggestive of a primitive neuroectodermal tumor. The tumor was markedly positive for vimentin (Fig. 2A) and CD99 (Fig. 2B). Staging workup with a positron emission tomography-computed tomography (PET-CT) image (Fig. 3A) demonstrated a large avid fluorodeoxyglucose (FDG) uptake at the level of the lower sacrum measuring 


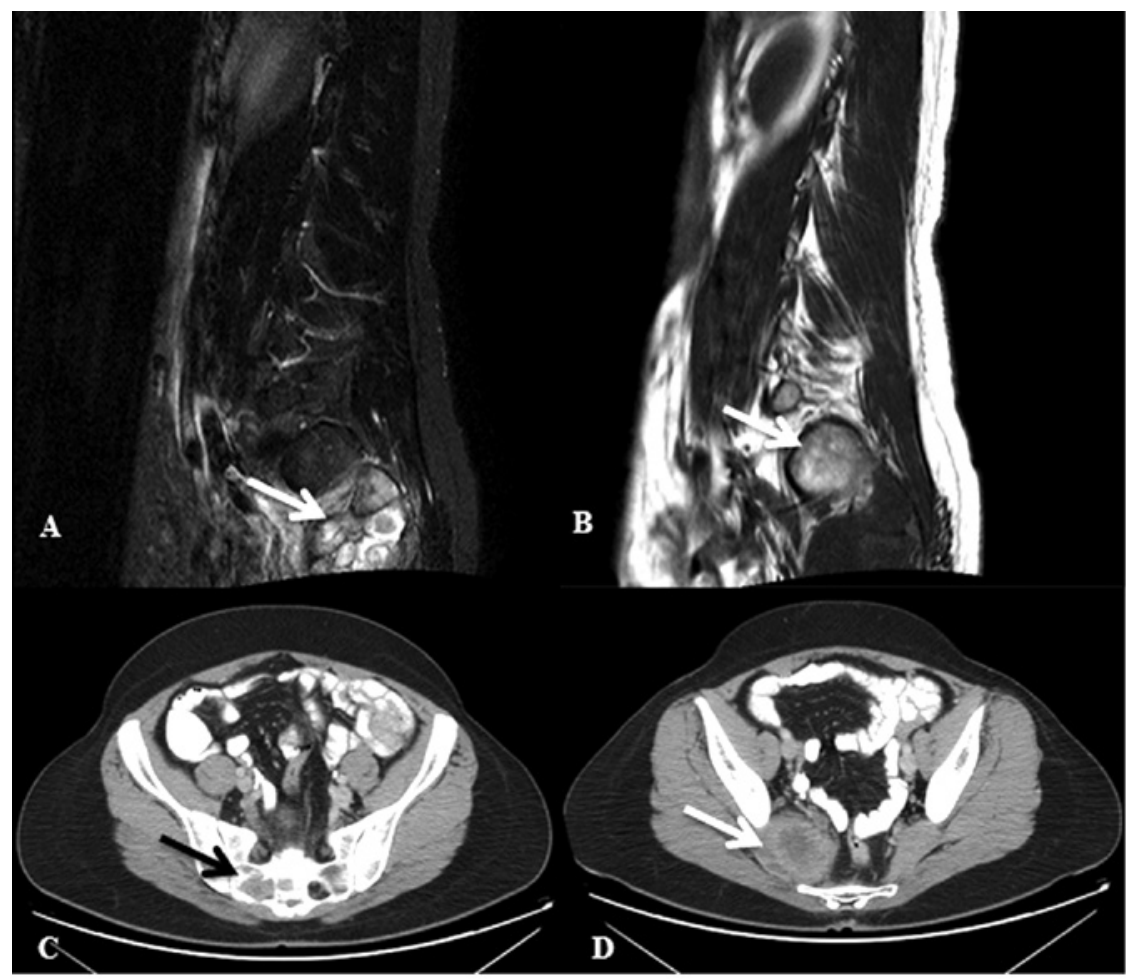

Figure 1. A lumbar spine MRI shows an irregular shaped right presacral mass (arrow) with (A) heterogeneous STIR hyperintensity and (B) T1 hypointensity. (C) CT of the pelvis shows a complex mass (arrow) with central necrosis and a thick enhancing wall extending into the right S2 neural foramen with lytic bone destruction. (D) Pelvic CT shows a 6x5x6 cm mass (arrow) compressing the right piriformis muscle posteriorly. CT, computed tomography.

\section{A}

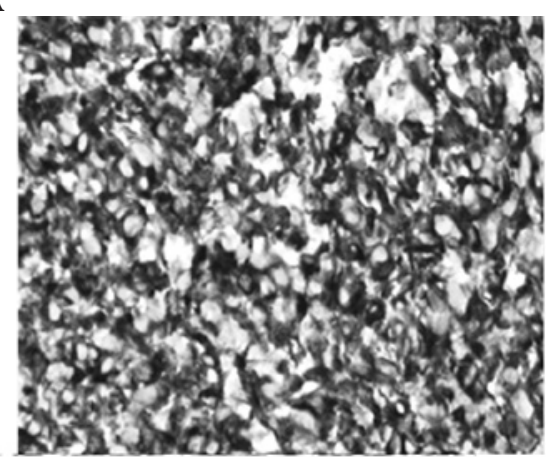

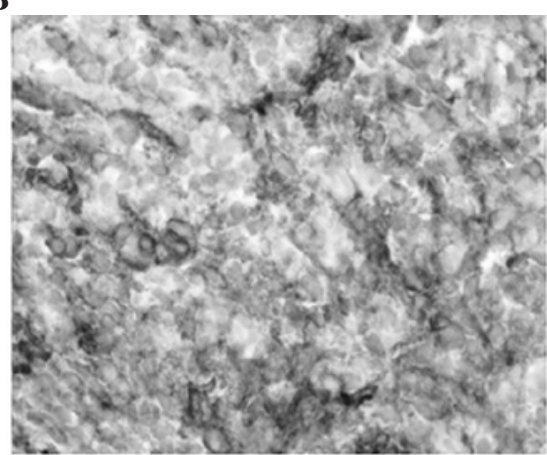

Figure 2. Tissue biopsy revealed a small, round blue cell neoplasm. Immunohistochemistry stains were positive for (A) vimentin and (B) CD99.

approximately $5.8 \mathrm{~cm}$. There was no other area of abnormally increased uptake of FDG in the whole body PET-CT. An open biopsy revealed a better preserved tumor with identical histological features testing positive for the ES translocation (22q12), and a diagnosis of ES stage III (T2bNOM0) was made.

The patient was treated with 17 cycles of vincristine $2 \mathrm{mg} / \mathrm{m}^{2}$, doxorubicine $75 \mathrm{mg} / \mathrm{m}^{2}$, cyclophosphamide $1200 \mathrm{mg} / \mathrm{m}^{2}$ with mesna rescue alternating with ifosfamide $1800 \mathrm{mg} / \mathrm{m}^{2}$ and etoposide $100 \mathrm{mg} / \mathrm{m}^{2}$ (IE) as described by Grier et al (6). Re-assessment after four cycles demonstrated a significant response. The involvement of S1 and S2 nerve roots represented a significant surgical challenge. As a result, the patient received external beam radiation at a dose of $56 \mathrm{~Gy}$ followed by 13 additional cycles of adjuvant chemotherapy. Post-treatment CT of the pelvis demonstrated a near complete resolution of the mass with minimal residual infiltrative soft tissue changes within the right presacral space. PET imaging demonstrated normalization of FDG uptake within the pelvic mass (Fig. 3B). Six weeks post-adjuvant chemotherapy, the patient presented with acute shortness of breath. PET-CT demonstrated an area of increased FDG uptake within the bilateral pulmonary hila, representing interval development of metastatic disease. A subsequent biopsy of the lung confirmed the diagnosis of metastatic ES. Two weeks later the patient succumbed to the disease after opting for comfort care.

\section{Discussion}

The mortality rate for ES is extremely high when treated with surgery or radiation therapy alone for local control of the disease (7). Over the past 5 decades, advances in chemotherapy, surgery and radiation therapy have improved the 


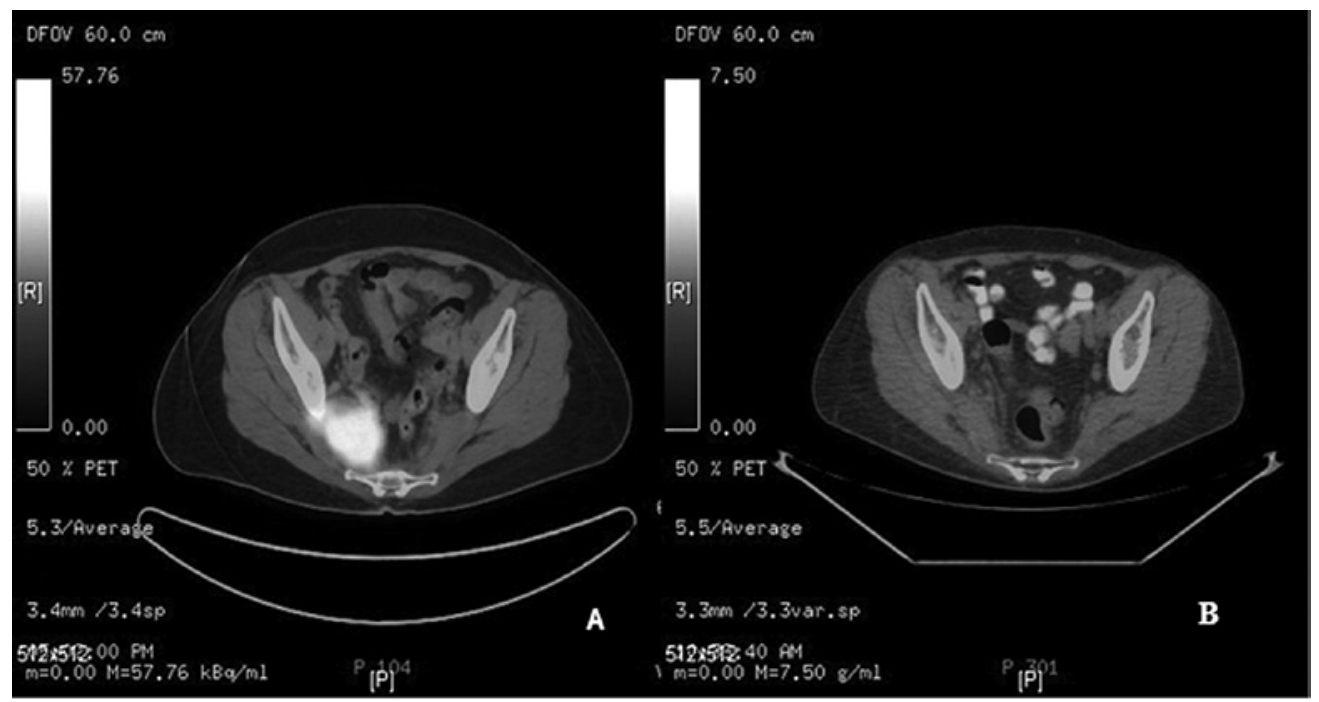

Figure 3. (A) PET-CT image at the level of the lower sacrum demonstrated a large avid fluorodeoxyglucose (FDG) uptake within the sacral mass measuring approximately $5.8 \mathrm{~cm}$. (B) Post-treatment PET/CT image shows normalization of FDG uptake within the pelvic mass. PET-CT, positron emission tomography-computed tomography.

prognosis of patients with ESFTs (8). The limited number of patients over the age of 40 and the exclusion of these patients from the majority of trials render these findings difficult to generalize (9).

Tumors of the pelvis have a poorer prognosis when compared with other sites. Whether this is related to the challenge of achieving local control or the proximity to critical deep structures remains to be elucidated. Current treatment recommendations are based on the available literature that is limited by selection bias, small study size, non-standard radiation therapy technique and lack of randomized trials comparing the two management approaches. The role of surgery in treating ES is controversial; however, certain studies suggest that resection with chemotherapy and irradiation positively impacts patient survival (10). Radiotherapy is usually applied at doses of 40-45 Gy for microscopic residues and 50-60 Gy for macroscopic disease (11). Treatment of adult patients follows the same principles. However, tolerability of therapies in adults are taken into account when transferring treatment protocols conceived for patients under the age of 30 years (11).

Although most cases of ES present as localized disease, overt metastases are capable of developing rapidly. Microscopic metastatic disease has been postulated to be present at the time of presentation. However, its spread is held in check by as yet unidentified factors secreted by the primary tumor. When the primary tumor is removed or irradiated, the loss of the putative suppressive factors may permit the metastases to grow. The use of chemotherapy in conjunction with surgery or radiation therapy to treat presumed metastatic disease has substantially improved survival (12).

Beyond specific clinical trials, patients with metastatic disease receive similar therapy to that administered for localized disease, with appropriate local treatment of metastases, usually radiotherapy. Certain studies have suggested benefit from intensive chemotherapy followed by autologous stem cell rescue, but randomized trials have not yet been performed and the benefit of stem cell transplant remains unproven. Patients with recurrent disease fare poorly, with 5-year survival rates of less than $20 \%$ (11). Current studies show that, following achieving remission in patients with non-metastatic ES, 30-40\% of these patients are likely to develop recurrence of local or metastatic disease (13). The majority of these studies report a time range of 2-10 years between commencing treatment and development of recurrence (14). Patients relapsing later than 2 years from initial diagnosis have more favorable outcomes (11).

This case report raises the importance of early metastasis in a treatment-responsive patient. According to current guidelines, initial work-up for staging in a non-metastatic ES is followed by reassessment of treatment response after 3-6 treatment cycles using focal PET-CT. In treatment responsive ES, local therapy is followed by additional chemotherapy. A surveillance follow-up imaging every 2-3 months for the first three years is recommended for localized, non-metastatic ES. The current case has demonstrated complex issues with localized pelvic ES in an older patient who initially responded well to chemo-radiation therapy, with complete resolution of the tumor. Despite adequate control of the local disease, multimodal therapy did not appear to affect metastasis. Although sarcomas are notorious for metastasis into lungs and bone, the utility of PET-CT and MRI for detecting subclinical recurrence or metastases has not been established during the treatment period. Only the primary site is evaluated by imaging for treatment response 10-12 weeks in to therapy.

In patients with lung metastases, the resection of residual metastases after chemotherapy, and whole lung irradiation, may grant a survival advantage (11). Most intensive therapies with additional agents have failed to markedly increase long-term survival in patients with metastatic disease. The impact of chemotherapy on metastasis of ES patients over the age of 30 remains to be elucidated. A recent intergroup study suggested that the addition of IE to traditional regimens may confer a local control benefit (15). Similar effects, with the addition of IE on survival benefit, have also been reported by another randomized trial among patients with non-metastatic pelvic ES (6). 
At present, patients should be offered participation in a clinical trial when available. Barring trial participation, multimodality therapy, as described above is recommended.

\section{References}

1. Grier HE: The Ewing family of tumors. Ewing's sarcoma and primitive neuroectodermal tumors. Pediatr Clin North Am 44 991-1004, 1997.

2. McManus AP, Gusterson BA, Pinkerton CR and Shipley JM: The molecular pathology of small round-cell tumours - relevance to diagnosis, prognosis, and classification. J Pathol 178: 116-121, 1996.

3. Stiller CA, Bielack SS, Jundt G and Steliarova-Foucher E: Bone tumours in European children and adolescents, 1978-1997 report from the Automated Childhood Cancer Information System project. Eur J Cancer 42: 2124-2135, 2006.

4. Leavey PJ, Mascarenhas L, Marina N, Chen Z, Krailo M, Miser J, Brown K, Tarbell N, Bernstein ML, Granowetter L, Gebhardt M, Grier HE and Children's Oncology Group: Prognostic factors for patients with Ewing sarcoma (EWS) at first recurrence following multi-modality therapy: a report from the Children's Oncology Group. Pediatr Blood Cancer 51: 334-338, 2008.

5. Athale U, Cox S, Siciliano S and Chan AK: Thromboembolism in children with sarcoma. Pediatr Blood Cancer 49: 171-176, 2007.

6. Grier HE, Krailo MD, Tarbell NJ, Link MP, Fryer CJ, Pritchard DJ, Gebhardt MC, Dickman PS, Perlman EJ, Meyers PA, Donaldson SS, Moore S, Rausen AR, Vietti TJ and Miser JS: Addition of ifosfamide and etoposide to standard chemotherapy for Ewing's sarcoma and primitive neuroectodermal tumor of bone. N Engl J Med 348: 694-701, 2003.

7. Nesbit ME: Ewing's sarcoma. CA Cancer J Clin 26: 174-180, 1976.
8. Indelicato DJ, Keole SR, Shahlaee AH, Shi W, Morris CG, Gibbs CP Jr, Scarborough MT and Marcus RB Jr: Impact of local management on long-term outcomes in Ewing tumors of the pelvis and sacral bones: the University of Florida experience. Int J Radiat Oncol Biol Phys 72: 41-48, 2008.

9. Bacci G, Balladelli A, Forni C, Ferrari S, Longhi A, Benassi MS, Briccoli A, Serra M and Picci P: Adjuvant and neoadjuvant chemotherapy for Ewing sarcoma family tumors in patients aged between 40 and 60: report of 35 cases and comparison of results with 586 younger patients treated with the same protocols in the same years. Cancer 109: 780-786, 2007.

10. Sciubba DM, Petteys RJ, Garces-Ambrossi GL, Noggle JC, McGirt MJ, Wolinsky JP, Witham TF and Gokaslan ZL: Diagnosis and management of sacral tumors. J Neurosurg Spine 10: 244-256, 2009.

11. Paulussen M, Bielack S, Jurgens H, Casali PG; ESMO Guidelines Working Group: Ewing's sarcoma of the bone: ESMO clinical recommendations for diagnosis, treatment and follow-up. Ann Oncol 20 (Suppl 4): 140-142, 2009.

12. Skubitz KM and D'Adamo DR: Sarcoma. Mayo Clin Proc 82: 1409-1432, 2007.

13. Bacci G, Ferrari S, Longhi A, Donati D, De Paolis M, Forni C, Versari M, Setola E, Briccoli A and Barbieri E: Therapy and survival after recurrence of Ewing's tumors: the Rizzoli experience in 195 patients treated with adjuvant and neoadjuvant chemotherapy from 1979 to 1997. Ann Oncol 14: 1654-1659, 2003.

14. Hanna SA, David LA, Gikas PD, Tindall AJ, Cannon SR and Briggs TW: Very late local recurrence of Ewing's sarcoma - can you ever say 'cured'? A report of two cases and literature review. Ann R Coll Surg Engl 90: W12-W15, 2008

15. TI Yock, M Krailo, CJ Fryer, Donaldson SS, Miser JS, Chen Z, Bernstein M, Laurie F, Gebhardt MZ, Grier HE, Tarbell NJ; Children's Oncology Group: Local control in pelvic Ewing sarcoma: analysis from INT-0091 - a report from the Children's Oncology Group. J Clin Oncol 24: 3838-3843, 2006. 